\title{
Static and dynamic characterization of biomedical polyethylene laser welding using biocompatible nano-particles
}

\author{
Annamaria Visco ${ }^{1,2,}{ }^{*}$, Cristina Scolaro ${ }^{1}$, Teresa Terracciano ${ }^{1}$, Roberto Montanini ${ }^{1}$, Antonino Quattrocchi ${ }^{1}$, \\ Lorenzo Torrisi $^{3}$ and Nancy Restuccia ${ }^{3}$ \\ ${ }^{1}$ Department of Engineering, University of Messina, C.da Di Dio, 98166 Messina, Italy \\ ${ }^{2}$ Istituto per i Processi Chimico-Fisici CNR -IPCF, Viale Ferdinando Stagno d'Alcontres, 37 - 98158 MESSINA \\ ${ }^{3}$ Dept. of MIFT, University of Messina, V.le F. S. D'Alcontres 31, 98166 Messina, Italy
}

\begin{abstract}
In this research, polymeric joints of Ultra High Molecular Weight Polyethylene (UHMWPE) sheets were realized and welded by a diode laser operating at $970 \mathrm{~nm}$ wavelength. One of the polymer sheet was doped, at different concentrations, with nano filler (carbon, titanium and silver nano-particles ) in order to enhance the absorption coefficient at the laser wavelength. Laser operated in repetition rate with a maximum pulse energy $100 \mathrm{~mJ}$, for times of the order of 1-60 s transporting the light trough a fiber with $300 \mu \mathrm{m}$ diameter. The laser light has been transmitted by the transparent first polymer and absorbed on the face of the second doped polymer. At the interface of the two polymer foils, $0.5 \mathrm{~mm}$ thickness each, the released energy induces melting, assisted by pressure, producing a fast and resistant welding. Single lap and double lap geometries have been performed and studied by means of mechanical static (shear stress) and dynamical analysis. Effect of the different particles nature on the mechanical features of the joints has been evaluated. Morphological observation of the jointed areas are presented and discussed. Joints could be useful in biomedical field for their special features.
\end{abstract}

\section{Introduction}

The growing development in many plastics industries and the increasing attention paid to the quality and aesthetics of products has defined new technological demands for components junction processes. Welds must be excellent in mechanical and aesthetic quality without residues in the welded area [1].

Among the plastic materials, polyethylene is a thermoplastic bio compatible material (for its chemical inertia), highly applied in the biomedical field [2,3]. For its high mechanical ductility and other important features, polyethylene is considered an unique material, used in several industrial sectors such as health and energy, and for advanced applications. Polyethylene is also a weld able polymer, and good quality joints can be obtained by employing diode or Nd:Yag lasers [4,5].

Mechanical characterization is a very important aspect of materials knowledge since polymers are often used in applications that involve stresses, such as that of polymeric joints. So we must study the effect that stress has on them. In the field of mechanical characterization, static testing (load/displacement curves) and fatigue test (lifetime or durability analysis) are commonly employed. In our case, the mechanical properties of our joints have been studied to verify the resistance features of the laser welding effect. In particular, mechanical static and dynamic (fatigue) tests have been performed. Life of polymeric and composite joints is an important and crucial aspect that needs attention. It has been researched in the last 40 years. Renton et al. [6] and Ferreira et al. [7] studied the fatigue behavior of polymeric and composite single lap joints, putting into evidence the effects of layer orientation, lap joint length and water immersion. In both papers, the failure appeared in the adhesive layer. The fatigue life is strongly influenced by the profile of the edges of the joint. Harris et al. [8] verified that the initiation of small cracks at the edges points represents the major part of the fatigue life. Moreover, temperature and humidity of the adhesive cure are extremely relevant for the damage accumulation. They produce significant changes in the stress state of lap joints [9].

In this paper we have considered a particular type of polyethylene, a biomedical grade Ultra High Molecular Weight-UHMWPE. It has a particular high viscosity and low friction coefficient, and it's largely used for biomedical applications [2].

Polyethylene itself is transparent to laser light. So, we have prepared single lap or double lap joints (welded by means of a diode laser) by employing fillers (nano particles) to make laser absorbing one polymeric sheet of the joint. The nanofillers have been suitably selected for type and quantity to keep color as close to white as possible for aesthetic requirement. The joints have been

\footnotetext{
Corresponding author: avisco@unime.it
} 
mechanically analyzed by means of static and fatigue tests in order to verify their strength and durability.

\section{Materials and Methods}

\subsection{Polymer/nanocomposite sheets preparation}

Polymeric nanocomposite sheets, $30 \mathrm{~mm}$ x $20 \mathrm{~mm}$ x 0,5 $\mathrm{mm}$ thick, were obtained by dispersing the carbon nanofiller, titanium dioxide and silver nano particles inside the matrix (biomedical grade UHMWPE GUR 1020) with a solvent.

The amount of fillers added to the UHMWPE polymer matrix was of 0.003-0.016-0.025-0.25-0.5-1.0\% (for carbon nanoparticles, UH-NC code), 1-2-4-6 \% (for titanium nanoparticles, UH-NTiO code) and $1-2-4 \%$ (for silver nanoparticles, UH-NAg code).

Polymeric nanocomposites of carbon are filled with very low filler amount of nanoparticles, $(0.003 \%, 0.016 \%$, $0.025 \%$, ) so that it anyway appears of white color.

The obtained nanocomposites were then molded into an hot press obtaining $60 \mathrm{~mm} \times 60 \mathrm{~mm}$ and $0.5 \mathrm{~mm}$ thick sheets. The preparation steps are shown in Fig.1.
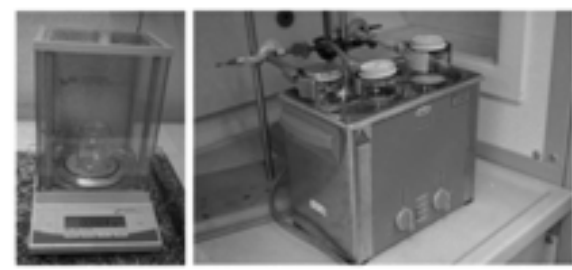

\begin{tabular}{|c|}
\hline Weight \\
UHMWPE \\
+ \\
Filler \\
\hline
\end{tabular}
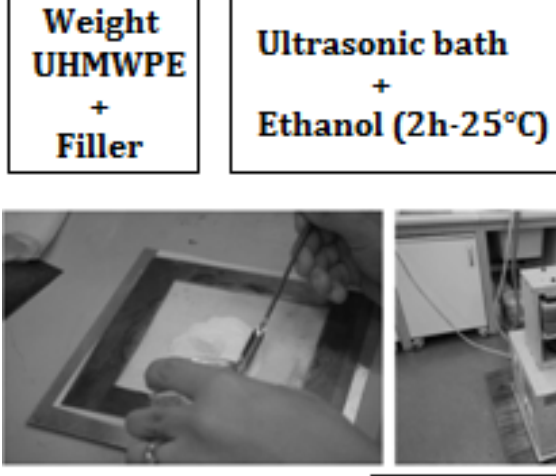

Mold

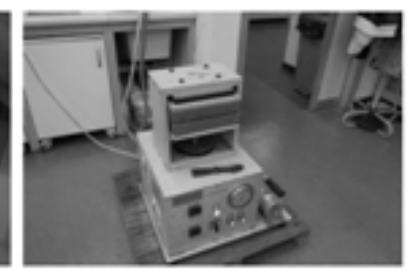

Compression Molding $\left(200^{\circ} \mathrm{C} / 20 \mathrm{~min}-200 \mathrm{~atm}\right)$
Fig. 1. Preparation of nanocomposite sheets

Single (SLJ) and double lap joints (DLJ) (Fig.2.a) were obtained by a diode laser operating at $970 \mathrm{~nm}, \mathrm{CW}$ continuous wave $(0.5-7 \mathrm{~W})$, maximum energy of $100 \mathrm{~mJ}$, for times of the order of 1-60 s. Optical fiber thickness: $300 \mu \mathrm{m}$.

Two types of welding geometry were created: points and grid (Fig.2.b). The irradiation time to create welding by a diode laser varies from a dozen seconds (NC-doped material) to over 2 minutes (doped with NAg and NTiO).

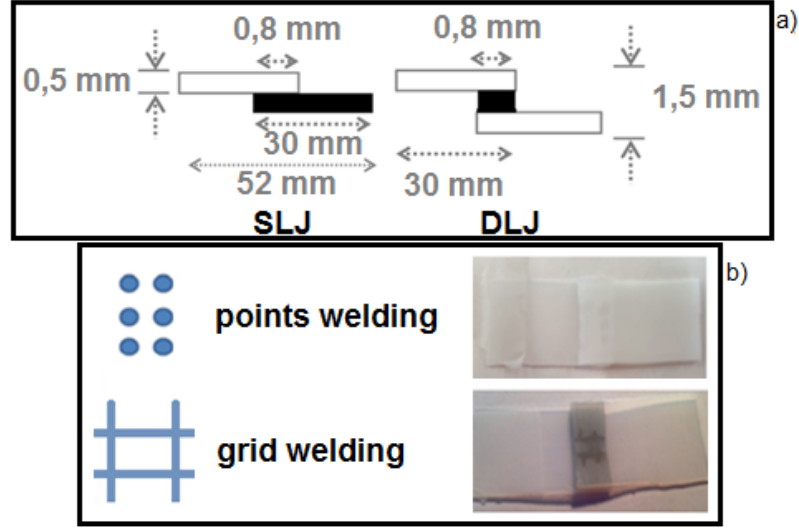

Fig. 2. a) Single-lap (SL) and double lap (DL) joint geometries; b) welding geometries and typical joint.

\subsection{Physical Characterization}

Optical absorption measurements: light absorption coefficients in pure and doped polymers with fillers were measured by using optical spectroscopy in the range of $150 \mathrm{~nm}$ to $800 \mathrm{~nm}$. Absorption studies were performed by evaluating the absorption of characteristic peaks emitted by a $\mathrm{Hg}$ (Ar) lamp and detected by the high sensitivity optical spectrometer.

The sample is placed on a suitable support, the incident light is perpendicular to the sample and the emerging light is measured in intensity with respect to the incident. The light beam emerging from the sample is captured by a fiber interfaced with the optical spectrometer (Horiba Jobin) that detects wavelengths between $220 \mathrm{~nm}$ and $1100 \mathrm{~nm}$.

The Lynear spectroscope software acquires 100 spectra per second and the average spectrum is then stored on a PC.

Absorption coefficients, $\mu$, have been experimentally calculated using the Lambert law:

$$
\mu=-(\Delta x) \ln \left(I_{i} / I_{o}\right)
$$

where $\Delta x=$ thickness, $I_{0}=$ incident laser intensity and $I_{T}$ $=$ transmitted laser intensity.

Morphological Optical (MO) characterization: the Hirox Digital Microscopekh KH8700 optical microscope has been used for morphological observation of the nanocomposite materials surface with a low magnification, of 50x.

\subsection{Mechanical Characterization}

Static tensile tests have been conducted to evaluate how the presence of $\mathrm{NC}, \mathrm{NTiO}$ and $\mathrm{NAg}$ at different concentrations in UHMWPE affects the mechanical properties of the final nanocomposite. The tests were performed according to the ASTM D638 standard with a Lloyd Universal Testing Machine (LR10K), imposing a traverse feed rate of $5 \mathrm{~mm} / \mathrm{min}$ and a load cell of $500 \mathrm{~N}$. Load/displacement curves of the joints shear stressed during the tensile motion. The data are from the average of six specimens. 


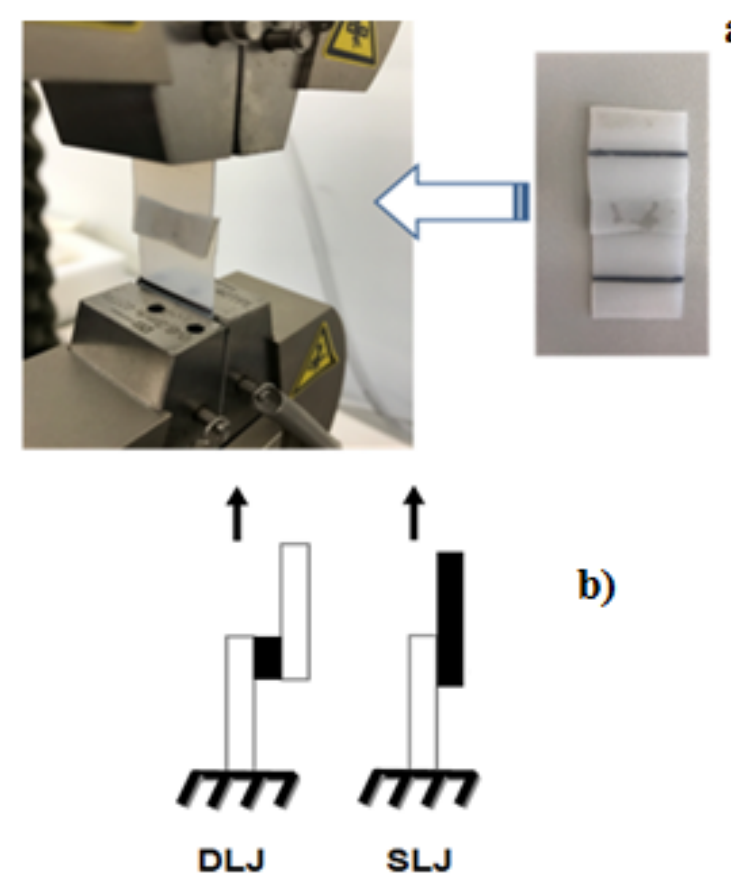

Fig. 3. a) Photograph of a joint and its disposition between the clamps during a mechanical tests and $b$ ) scheme of double and single lap joint's arrangement during the test .

Dynamic fatigue tests: the experimental setup allows to test the sample with fatigue loads, monitoring the mechanical and thermal behaviors. It consists of an electro mechanic testing system (Electropuls E300, Instron), equipped with two pneumatic grips $( \pm 3 \mathrm{kN}$ max) able to guarantee a grip force of $4.5 \mathrm{kN}$, a calibrated load cell $( \pm 5 \mathrm{kN} \max )$, and an infrared camera (SC 7200, Flir). The fatigue tests were performed according to ASTM D7791 standard. They were carried out in load control at different maximum force values (fractions of the break load) with stress ratio (R) of 0.7 and a frequency of $1 \mathrm{~Hz}$ at room temperature $\left(25 \pm 2{ }^{\circ} \mathrm{C}\right.$ and $50 \pm 10 \mathrm{RH})$. The mechanical parameters were acquired at $100 \mathrm{~Hz}$ with a defined reduction of the analyzed cycles: from 0 to 1000 th cycle all ones were captured, between 1000th and 100000th cycle 1 every 4 , finally after 100000th 1 every 40. IR images were obtained at an acquisition frequency of $100 \mathrm{~Hz}$, monitoring the thermal effect at the maximum applied force of the characteristic cycles and assuming as negligible the small variation of the temperature room $\left( \pm 2{ }^{\circ} \mathrm{C}\right)$ during the test.

\section{Results and discussion}

The presence of filler within the UHMWPE makes the nanocomposites less transparent to the laser radiation. Increasing the percentage of filler concentration the absorbance increases at the laser wavelength in $0.5 \mathrm{~mm}$ thick target. Pure polyethylene (UHMWPE) instead is a transparent material to laser light.
Fig.4.a shows the variation of light absorption coefficient in pure and doped polymers at different percentages by weight $(0.003 \%, 0.016 \%, 0.025 \%$, $0.25 \%, 0.5 \%, 1 \%)$ of nano carbon particles. From Fig.4.b we observe how the light absorption coefficient in the polymer, for example, at $545 \mathrm{~nm}$, grows with the growth of the amount of carbon nanostructures. A similar result has been obtained testing the other fillers.
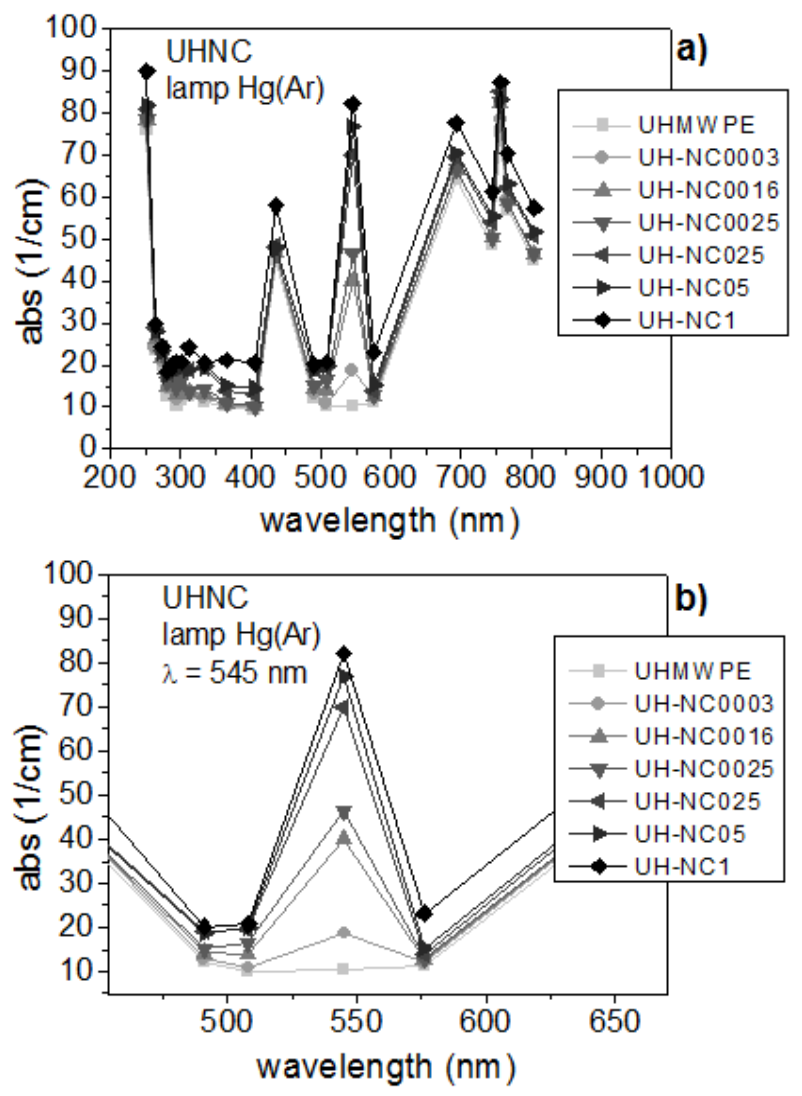

Fig. 4. a) Measure the absorption coefficient of pure polyethylene with doped with $\mathrm{NC}, \mathrm{b}$ ) measure the absorption coefficient at $\lambda=545 \mathrm{~nm}$ according to the NC filler percentage.

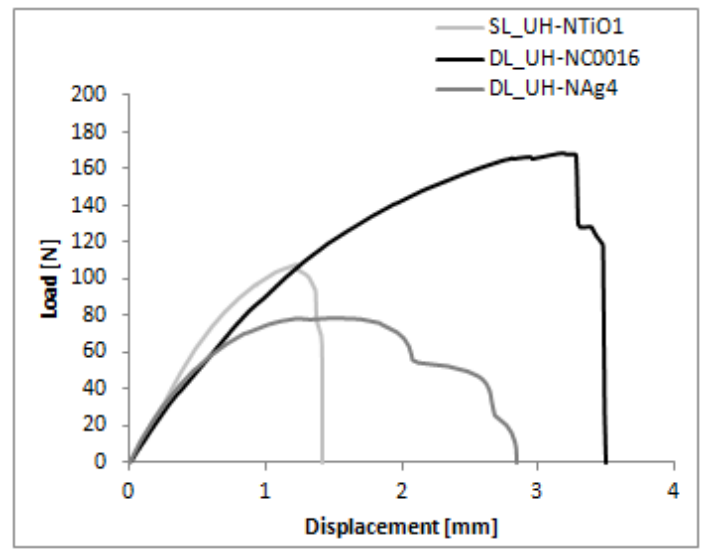

Fig. 5. Load /displacement curves of the joints with different filler types.

Regarding the static mechanical tests of the prepared joints, a previous experimentation (not shown for brevity) indicated to us that the optimal filler amount was of $0.016 \mathrm{wt} \%$ of $\mathrm{CN}, 1 \mathrm{wt} \%$ of $\mathrm{TiO}_{2}$ and of $4 \mathrm{wt} \%$ 
of Ag. The static load/displacement curves shown as the highest load of $169 \mathrm{~N}$ is reached in the $\mathrm{NC}$ filled joint and the lowest of $78 \mathrm{~N}$ in the Ag filled one (Fig.5). The values of static strength of these joints are anyway appreciable, compared to the mechanical behavior of other similar polymeric joints [2]. In Table 1 are listed the contact angle values measured in water and the surface roughness of above mentioned nanocomposites. Values indicated a more hydrophobic character of the materials filled with Ag and less in these filled with $\mathrm{TiO}_{2}$ and $\mathrm{NC}$, while surface roughness was within the range 2.0-3.3 micron.

Table 1. Mechanical and physical features of the joints.

\begin{tabular}{|c|c|c|c|c|c|}
\hline \multicolumn{7}{|c|}{ Mechanical statical test results and surface features } \\
\hline Joint code & $\begin{array}{c}\text { Filler } \\
\text { amount } \\
(\%)\end{array}$ & $\begin{array}{c}\text { Load } \\
(\mathbf{N})\end{array}$ & $\begin{array}{c}\text { Displacement } \\
(\mathbf{m m})\end{array}$ & $\begin{array}{c}\text { Contact } \\
\text { angle } \\
\left({ }^{\circ}\right)\end{array}$ & $\begin{array}{c}\text { Roughness } \\
(\mu \mathrm{m})\end{array}$ \\
\hline UH-TiO $_{2}$ & 1 & 107 & 2.76 & 94 & 2.00 \\
\hline UH-NC & 0.016 & 169 & 3.49 & 93 & 2.09 \\
\hline UH-Ag & 4 & 78 & 2.16 & 77 & 3.31 \\
\hline
\end{tabular}

The morphological observation of the welded area of the UH-NC0016 joint (that exhibited the best static mechanical strength) is shown in Fig.6. The image highlights the melted track with the cross line shape. The laser contact with the polymer melts the materials, changing the starting smooth morphology of polyethylene surface.
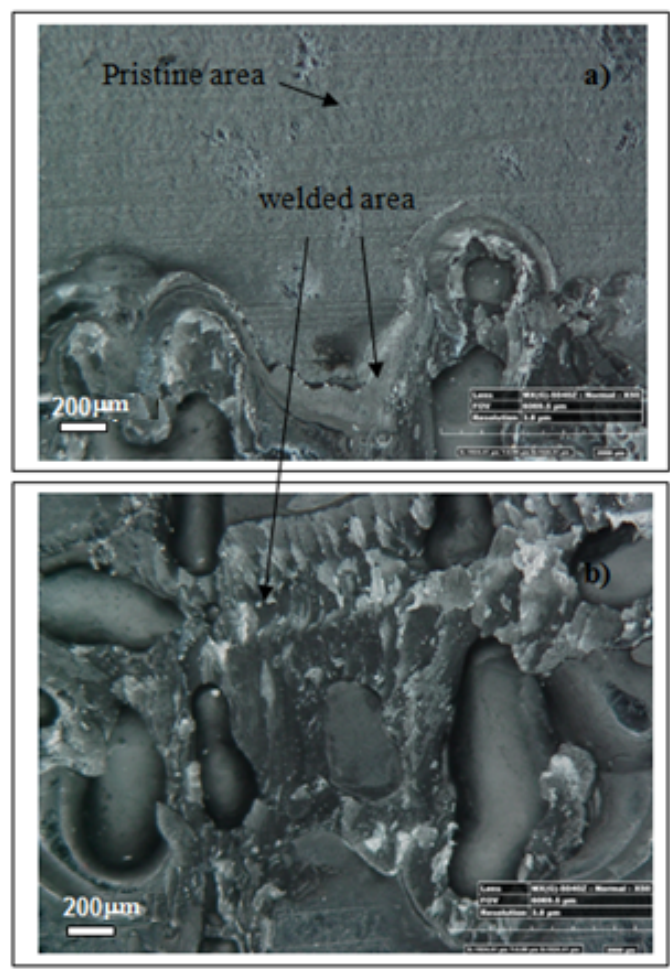

Fig. 6. Microscope images of UH-NC0016 welding junction a) of the pristine polymeric surface and b) of the laser irradiated surface.

The great morphological modification indicates a deep interpenetration between the polymeric sheets confirming a good welding action, in agreement with mechanical results. Fig.7 highlights the displacement of the fatigue machine actuator, and so the elongation of two welded joints of UH-NC0016 sample, subjected to $90 \%$ of break load (FR). Three different regions can be identified by observing the displacement-number of cycles trend:

(1) a first part with elastic deformation due to polymeric structure orientation for the normal load;

(2) a second step with stiffening of the basic material of the joints and progressive plastic deformation;

(3) finally, a rapid damage and a break of the welded zone.

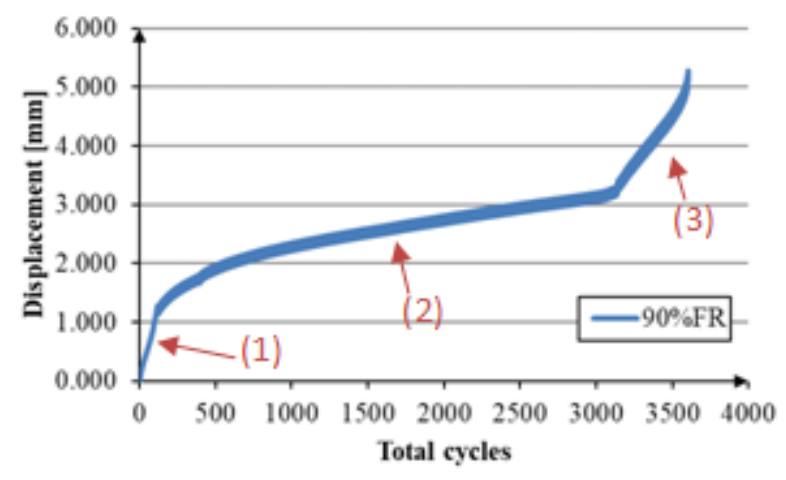

Fig. 7. Elongation of two welded joints

The fatigue limit of the UH-NC0016 joint is about 3500 cycles. Fig. 8 shows the typical images of the thermal behaviors of the cyclic load at $90 \% \mathrm{FR}$, when the sample is subjected to the maximum load $\left(\mathrm{F}_{\max }=130 \mathrm{~N}\right)$ on the fatigue test.

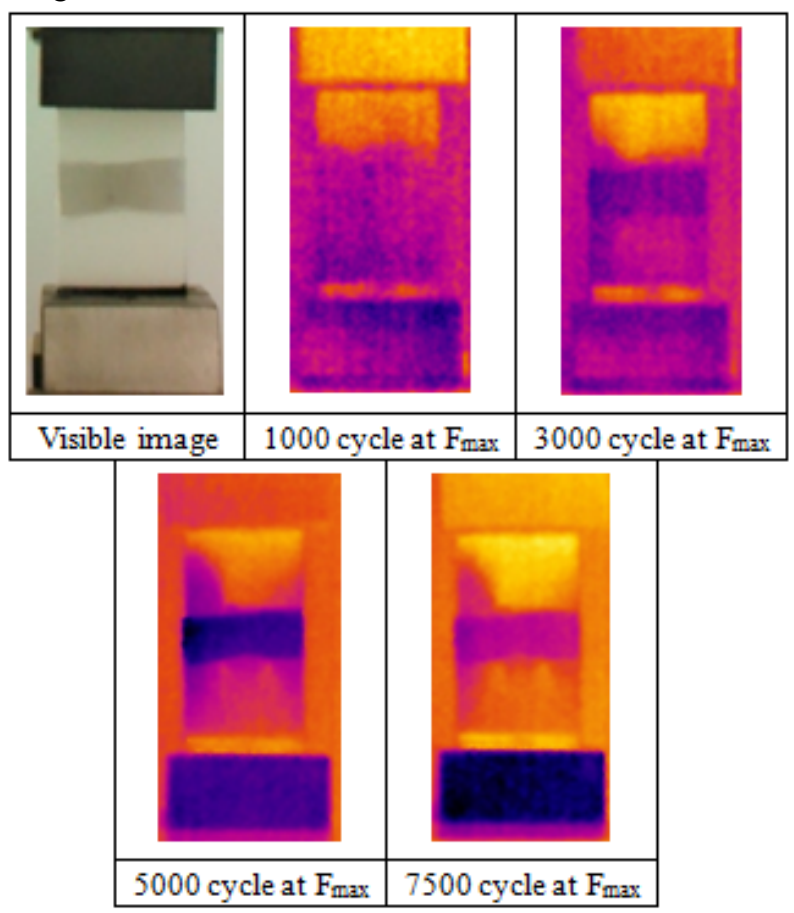

Fig. 8. Comparison between visible image and IR images at maximum load and specific cycles

In this condition, the thermal increase starts to the upper joint, locked at the machine fatigue actuator. Progressively, it evolves to the inferior joint and to the 
welded zone. Particularly, in the welded zone, the temperature growth is only placed closer to the melted area.

Fig.9 displays the effect of the applied load on the measured elongation of the two welded joints. Different trends for compression and tensile are observed, defining a hysteric cycle. The hysteresis area grows when the number of fatigue cycles increases from 50 cycles (Fig.9.a) to 3500 cycles (Fig.9.c), putting into evidence the plastic deformation of the joints whose displacement improves from $\sim 1.45 \mathrm{~mm}$ (Fig.9.a), to $\sim 2.65 \mathrm{~mm}$ (Fig.9.b) and to $\sim 4.7 \mathrm{~mm}$ (Fig.9.c).
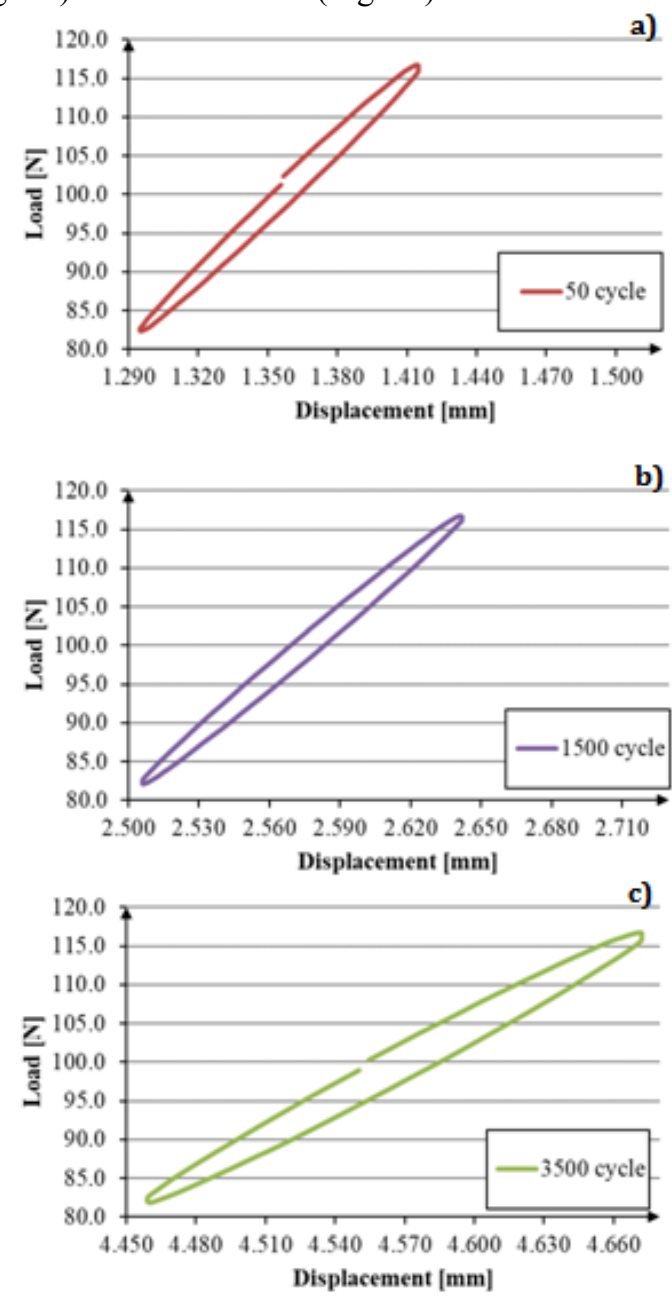

Fig. 9. Areas of hysteresis at $90 \% \mathrm{FR}$ and different cycles a) 50 , b) 1500 , c) 3500 .

\section{Conclusions}

Polymeric sheets were welded in two different geometries by means of a diode laser obtaining single lap and double lap SL joints. One of the sheets must be doped with an absorbent filler in order to absorb the laser energy, melting the overlapped sheets at their interface. Polymeric nanocomposites are filled with very low filler amount, (carbon, titanium dioxide and silver nanoparticles) so that it anyway appears of white color. This can be useful for aesthetic reasons. Melted area create a strong joint that exhibits a high mechanical resistance, during both static and fatigue tests.
The highest shear load has been shown by the 0.016 weight $\%$ of NC filled nanocomposite $(169 \mathrm{~N})$. Its fatigue cycles resistance (at $90 \%$ of maximum load) were of about 17000 while at $70 \%$ of maximum load were about 350 . The other joints made by the nanocomposites filled with 1 weight $\%$ of titanium dioxide and 4 weight $\%$ of silver nanoparticles, reached a lower strength of $107 \mathrm{~N}$ and $78 \mathrm{~N}$, respectively, due to a lower filler absorption power and, consequently, to a lower adhesion at the polymeric sheets interface. Experimental results performed with the dynamic fatigue test coupled with thermography camera, resulted successfully applicable in order to check the fatigue behavior and life of this type of polymeric joints, giving a good response during the tests. In fact fatigue tests highlighted an appreciable mechanical behavior of the polymeric joints: in particular, the fatigue limit of the UH-NC0016 joint (subjected to $90 \%$ of break load) was about 3500 cycles. Three different steps for damage of the single lap joints have been identified: elastic deformation for the basic material of the joint, plastic deformation of the sample and rapid damage and break for the welded zone. Hysteresis and IR images have been confirmed the previous observations: single lap joints failure is mainly due to the damage of the welded zone.

This joint exhibits appreciable mechanical and aesthetic features; next step will be the biomedical features evaluation of the materials in order to consider the possible biomedical applications for medical device technology, human care or domestic appliances (such food and beverage packaging).

This work was supported by the "Research and Mobility" project of Messina University No. 74893496, scientifically coordinated by Professor L. Torrisi.

\section{References}

1. S.T. Amancio-Filho, J.F. dos Santos, Polymer Engineering and Science, 49, 1461-1476, (2009).

2. G. Galtieri, A. Visco, D. Nocita, L. Torrisi, G. Ceccio, C. Scolaro, PPLA 2015, Journal of Scientific Instruments, 11 C04013, (2016), DOI 10.1088/1748-0221/11/04/ $\overline{\mathrm{C}} 04013$.

3. A.Visco, V. Brancato, L. Torrisi, M. Cutroneo, Int. J.Polym. Anal. Charact. 19:489-499, (2014).

4. A.Visco, G. Galtieri, L. Torrisi, C. Scolaro, Int. J.Polym. Anal. Charact. 20:442-456, (2015).

5. L.Torrisi, C. Scolaro, N. Restuccia, J Mater Sci: Mater Med, 28(4):28-63, (2017), DOI 10.1007/s10856-017-5871-1.

6. W.J. Renton et al., J Aircraft, 12(5:):442-7, (1975).

7. J.A.M. Ferreira et al. Composites Science and Technology 62, 1373-1379 (2002)

8. ] Harris JA, Fay PA., Int J Adhes Adhes, 12(1:):918, (1992).

9. Adams RD, Coppendale, J, Mallick, V, Al-Hamdan H, Int J Adhes Adhes, 12(3):185-90 (1992). 